\title{
RGB image de-noising Using New Low-pas filter with variable Gaussian core Real time optimized by Neural networks
}

\author{
Tebini Sondes, Seddik Hassene,Mbarki Zouhair and Ben Braiek Ezzedine \\ tebinisondes@yahoo.fr, hassene.seddik@estt.rnu.tn and ebenbraiek@yahoo.fr
}

\begin{abstract}
Filtering consists in applying a non linear transformation on the image intensities by convolution to modify its characteristics. Gaussian filter is widely used in literature as a low pass filter for signal de-noising. It has some advantages and many inconvenient. It presents a static shape that convolves uniformly the entire image zones. Its smoothing efficiency depends on the value of its standard deviation. More its smoothing efficiency is increased more the image is blurred and the details and borders are removed. All these inconvenient are related to the static nature of Gaussian core of the filter. In this paper we propose a new approach for RGB images filtering, based on a smart dynamic filter with variable Gaussian core based neural network. The parameters that intervene in the filtering process are real time computed and supervised by a neural network. The filter is continuously varied to detect and clean noisy zones and avoid clean zones in the image. The experimental results demonstrate the efficiency of the proposed technique. The image is well filtered and the details and borders are more conserved.
\end{abstract}

Keywords-efficient RGB image denoising, neural networks, optimization, parameter estimation,peak signal to noise ratio.

\section{INTRODUCTION}

2D-Filtering is a mathematical process applied on the image matrix. Many applications are related to this domain such as noise reducing, edges extracting... Removing noise from the original signal is still a challenging problem for researchers. There have been several published algorithms and each approach has its inconvenient and advantages. There are two basic approaches to image denoising, spatial filtering methods and transform domain filtering methods. The most known inconvenient of spatial method filtering is the blurring of the image and removing some data. All these inconvenient are related to the static nature of the filter. There have been a number of methods developed for image denoising. Median filtering and bilateral filtering [1] are able to reduce noise, as well as preserve edges. However, these methods tend to lose textures. Some color image denoising filters are reported in [2, 3 and 4] in order remove the undesired effect. Most of the denoising techniques available in literature are developed and tested only for gray images [5, 6 and 7]. In recent past, a few color image denoising filters are reported in the literature $[8,9$ and 2]. In [10] we found a new method of adaptive filtering based on a multichannel least-squares lattice filter to restore degraded color images in the RGB color system. The basic objectives of the preprocessing of the color image are the same as the mono-components image in order to remove noise preserving the image features [11 and 15]. In [16] multichannel versions of a circular spatial filter are proposed and developed. It is difficult to reduce color noise at high speed without losing image details. To overcome this problem, a new denoising algorithm for color images is described in [17]. Spatial filters and especially gaussian filter is a way to remove noise to a reasonable extent but at the cost of blurring images which in turn makes the edges in pictures invisible. This inconvenient is related to the static nature gaussian core of the filter, that's why it is necessary to apply an efficient denoising technique to compensate for such data corruption. The performance of our approach is carefully examined and compared to the classical method for grayscale images[18] and performed very well for efficient suppression of not only additive noise but also of multiplicative once . Experimental results on synthetic images will illustrate the capabilities of the studied approach for RGB color image. The outline of the paper is as follows. In the next section, the improved neural networks optimization of variable gaussian filter for efficient RGB image de-noising is presented. Section 3 is devoted to qualitative evaluation of the results .Finally, concluding remarks are given in section 4 .

\section{THE PROPOSED APPROACH}

During the processes of acquisition and transmission, images are often contaminated by noise. That's why noise reduction is a difficult subject of image processing which is a fundamental tool in the context of the restoration of noisy color images. Many methods have been devoted in this field for example denoising by static Gaussian filter. It remove noise but produces some inconvenient like the blur and the loss of the important characteristics of the image which are related to its static nature. In this task, we create a variable gaussian filter in order to preserve the edges and conserve a good image quality.

\section{A. Color Image}

Color images are considered as three band monochrome images, where each band is of a different color. Each band 
provides the brightness information of the corresponding spectral band. The most widely used digital images are RGB (red, green and blue) mode [19] (Figure.1). Now, the visual information transmitted in the form of color image is becoming a major method of communication but the image obtained after some processing steps is often corrupted with noise so we refer to image denoising to produce a visually high quality image.

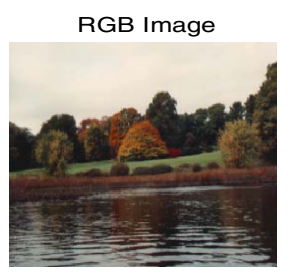

G Component

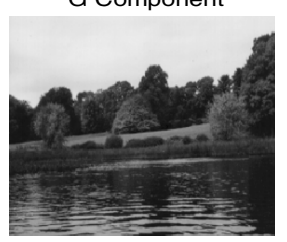

Figure 1. RGB image

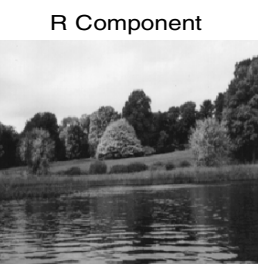

B Component

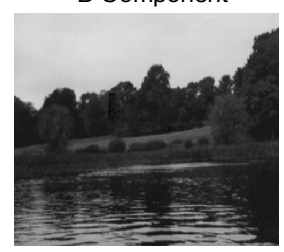

to an acceptable value. The outputs of this interface are the synaptic weights and the bias.

\section{Backpropagation Algorithm}

The most common approach to minimize the function $\mathrm{E}$ is shown in equation 2 .

$$
E=\frac{1}{2} \sum_{p=1}^{P} \sum_{k=1}^{K}\left(d_{p, k}-y_{p, k}\right)^{2}
$$

Weights update at time $\mathrm{k}+1$ contains the momentum of the previous updates $(\mathrm{k})$ by following equation:

$w_{k i}(t+1)=w_{k i}(t)+\Delta w_{k i}(t)$

Where

$P$ is the number of inputs and $k$ the number of outputs.

$d_{p, k}$ is the desired output, $y_{p, k}$ is the summing output.

\section{E. Principle of the Proposed Aproach}

The development of the algorithm including neural networks is done in three phases. The first phase is learning. Wherein, the network is driven from drive vectors. The second phase is the test; it is initiated when the network has finished learning. The third is an evaluation phase of our method which is the filtering by a Gaussian filter whose parameter $\sigma$ is optimized using the neural network, relative to the static method of filtering by Gaussian filter whose parameters are fixed. Our proposed optimization method is summarized as follows:

Step1: add noise to the image and segment it into 3 homogenous areas. Noise is present in an image either in an additive or multiplicative form (equations 4and 5).

$Y(x, y)=S(x, y)+N(x, y)$

$Y(x, y)=S(x, y) \times N(x, y)$

Where $\mathrm{S}(\mathrm{x}, \mathrm{y})$ is the original image, $\mathrm{N}(\mathrm{x}, \mathrm{y})$ denotes the noise introduced into the signal to produce the corrupted image $\mathrm{Y}(\mathrm{x}, \mathrm{y})$ and $(x, y)$ represents the pixel location.

Step2: fragment these areas in windows of the same size.

Step3: filter these windows with different Gaussian filters and meet the optimal variance for each window.

Step4: learning the neural networks (whose input is the noisy image and optimum sigma (variance) as the desired output).

Step5: testing the neural networks in order to determine the optimal vector sigma with which we will filter the noisy image.

Step6: Filter the noisy image with a static Gaussian filter and filter it by using the same variance as the output's neural networks.

Step7: Compute the corresponding PSNR (peak signal to noise ratio). 


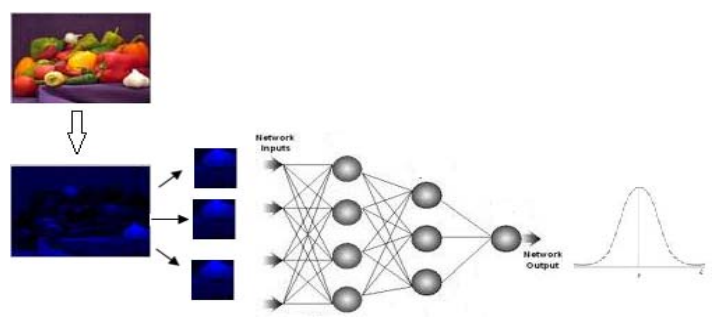

Figure 2. Neural network training

III. RESULTS AND DESCUSSION

In this section, we show the results of Filtering image by the supervised gaussian filter, and then evaluate the performance of the neural networks optimization. Among the variety of measures used, one measure commonly used is the peak signal to noise ratio (PSNR). This measure is used to quantify the distortion between two images using the following formula:

$P S N R=10 \cdot \log _{10}\left(\frac{d^{2}}{M S E}\right)$

Where

$M S E=\frac{1}{M \times N} \sum_{i=0}^{M-1} \sum_{j=0}^{N-1}\left([f(x, y)-r(x, y)]^{2}\right)$

MSE is the mean square error, it is defined for 2 images $f$ (original) and $\mathrm{r}$ (filtered image) and $\mathrm{d}$ is the image dynamic. For color images, the PSNR is calculated on each of the three color matrices and an average is then performed to obtain a final value.

\section{A. Salt \& Pepper Noise}

Salt and pepper noise is an impulse type of noise, which is also referred as intensity spikes. It satisfies the rule (equation 2). We chose to demonstrate the efficiency of the proposed technique based neural network by applying it for the Blue matrix as shown in Figure 2. This is due to the human sensitivity that is higher with the blue channel than with the green and the Red ones
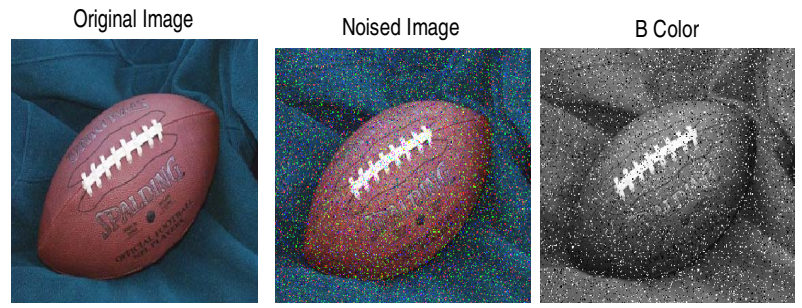

Figure 3. Test Image

After testing the network with different windows sizes, we found that the optimum window to segment the noised image is $8 \times 8$. We varied the intensity of the noise from 0.01 to 0.06 and we obtained the results of PSNR represented in Figure 3.

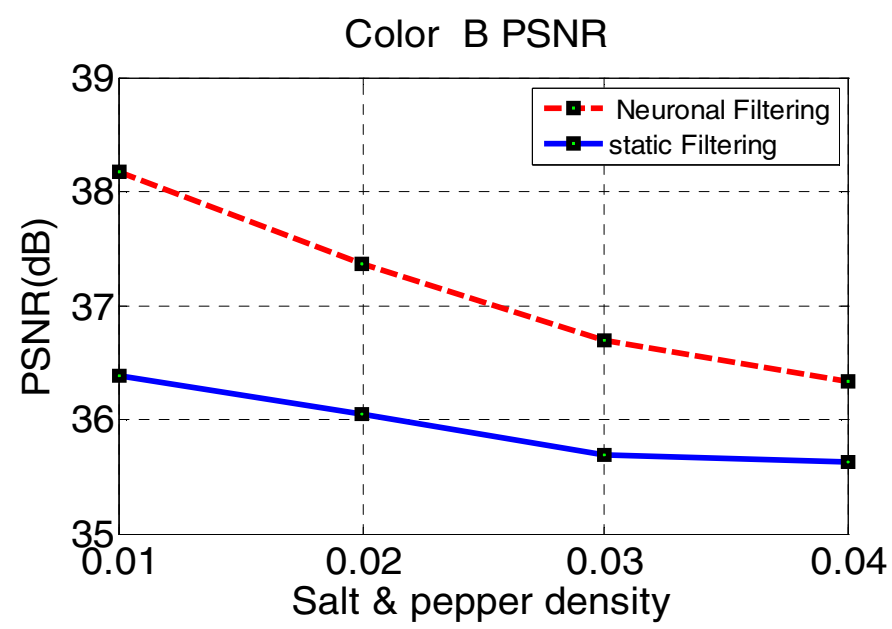

Figure 4. PSNR of the image corrupted by low density of Salt and Pepper noise

For the high densities of noise,we obtained this results of PSNR and corrélation shown in Figure 4 and 5.

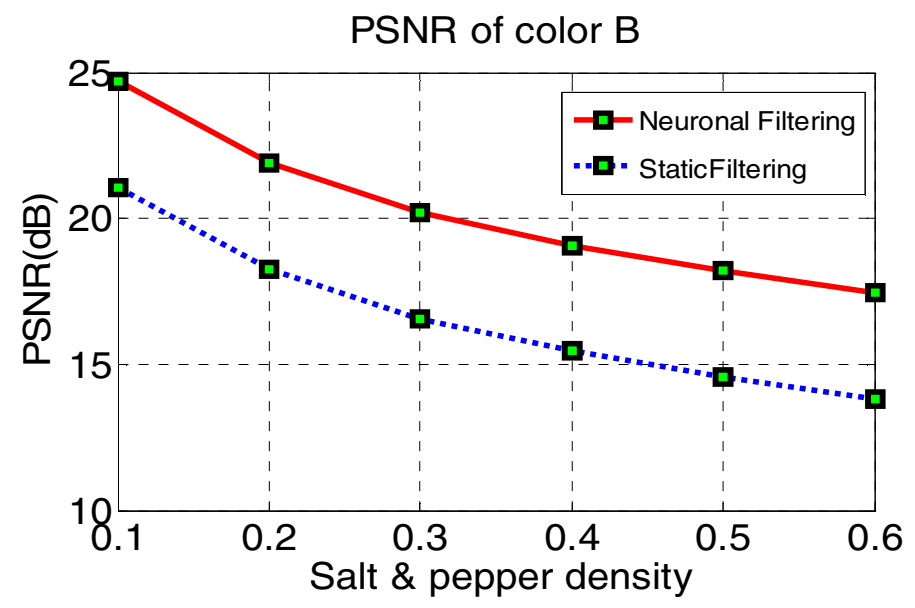

Figure 5. PSNR of the image corrupted by high density of Salt and Pepper noise

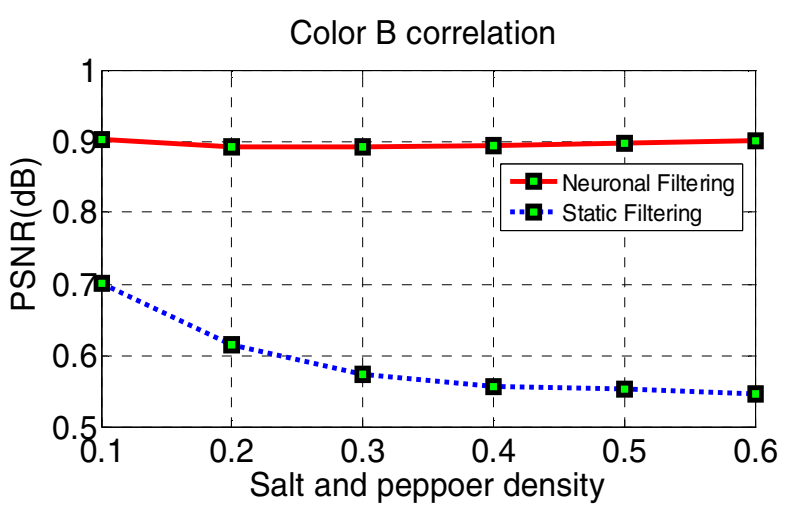

Figure 6. Correlation values between the original image and the filtred one 
All these figures demonstrate the efficiency of our neuronal method to supervise the gaussian filter, indeed, when we increase the density of the salt and pepper noise, and we observe that the variation of PSNR is better than the PSNR calculated after static gaussian filtering.

\section{B. Gaussian Noise}

It is an additive noise follows the rule (2).It is defined by its mean $(\mu)$ and its variance $(\sigma)$. We find that our neural approach for image filtering becomes even more efficient compared to the classical method, the ratio PSNR is important. Thus the result of the correlation between the original image and the noisy is very important, which explains the high quality of filtering. These figures illustrate the results of filtering the segmented image in windows of $64 \times 64$.

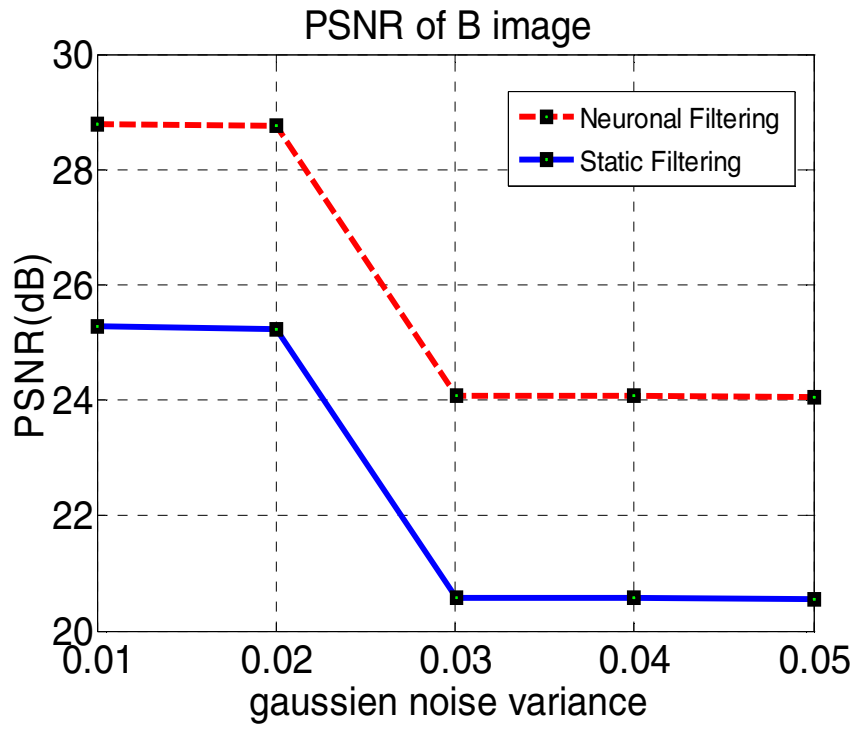

Figure 7. B image's PSNR

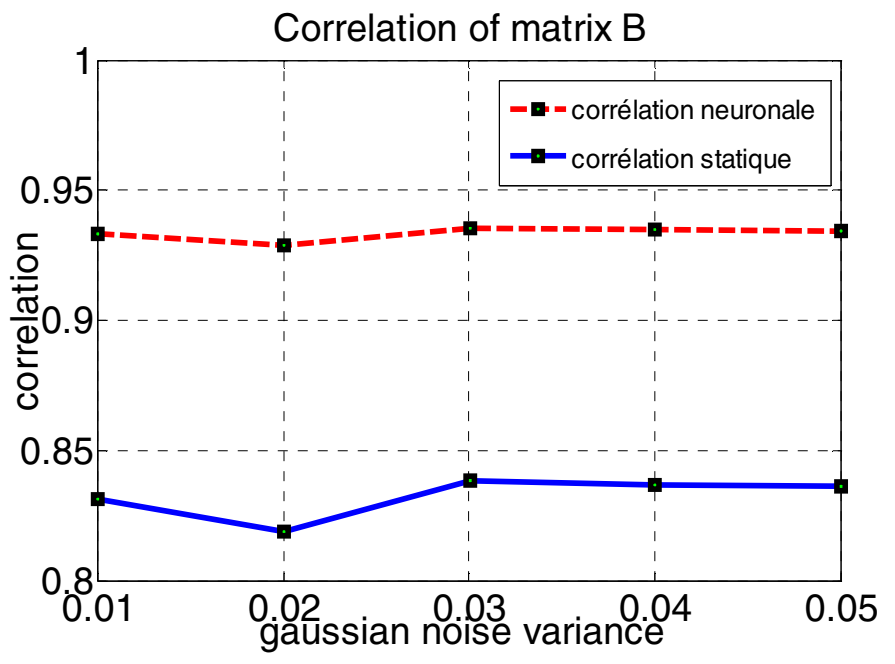

Figure 8. Correlation Results

\section{Speckle Noise}

The Speckle is a multiplicative noise (equation 5). Speckle is a multiplicative noise. This type of noise occurs in almost all coherent imaging systems such as laser, acoustics and SAR (Synthetic Aperture Radar) imagery. We will apply our method to the RGB test image bellow. We noted that the image was decomposed in windows of $16 \times 16$.
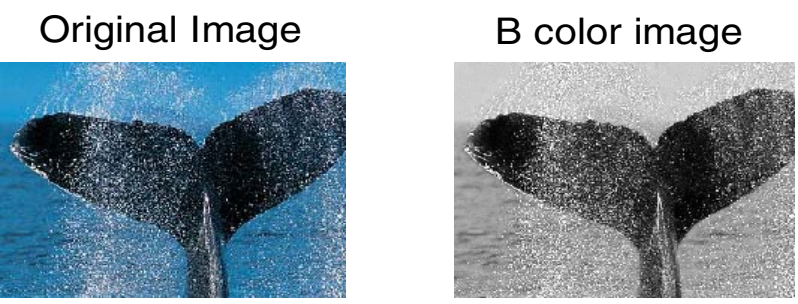

Figure 9. Test Image

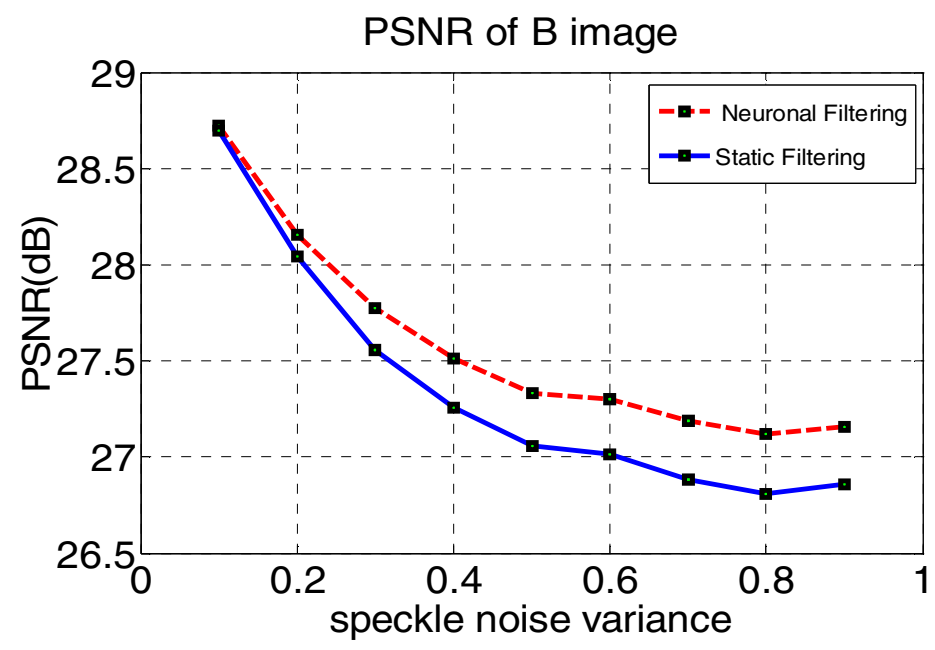

Figure 10. Speckle denoising results

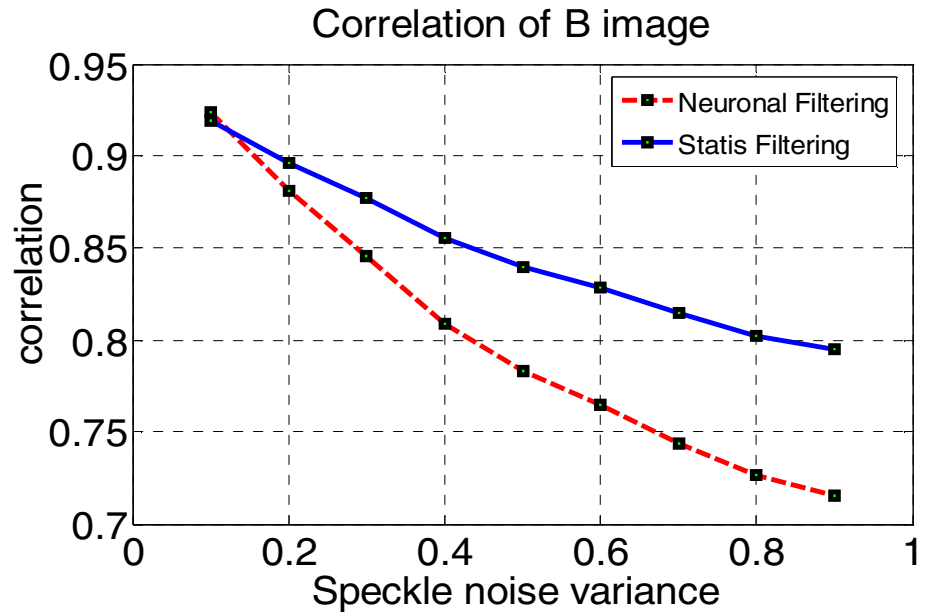

Figure 11. Correlation denoising results 
These figures show that the approach to optimize the Gaussian filter parameters gives a higher quality filtered image. even for very high noise densities.

In the next section, we will apply our neuronal approach by processing the R, G and B matrix. In this step the PSNR is calculated on each of the three color levels and an average is then performed to obtain a final value of RGB image. The processing steps are represented as follow:
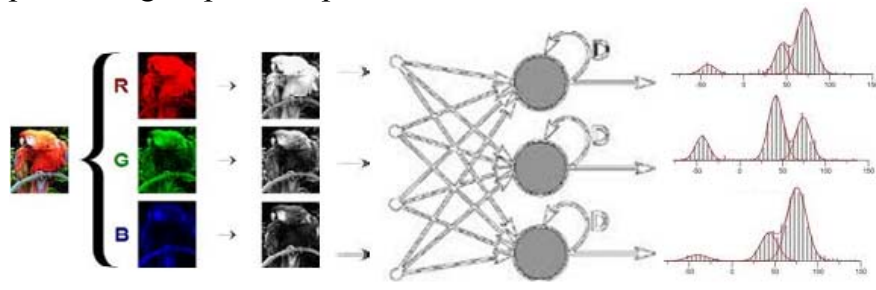

Figure 12. Neural network Processing

The image used to test our approach is represented in the next figure.
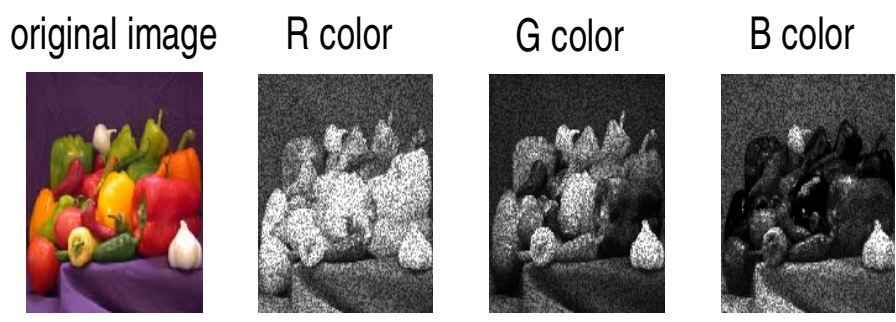

Figure 13. RGB Test Noised Image

The table bellow will demonstrate the efficiency of the proposed technique.

TABLE I. PSNR OF EACH COLOR MATRIX

\begin{tabular}{|c|c|c|c|c|c|}
\hline \multicolumn{2}{|c|}{} & \multicolumn{4}{c|}{ Salt \& Pepper Noise density } \\
\hline \multirow{2}{|c|}{ Color Matrix } & $\mathbf{0 . 0 1}$ & $\mathbf{0 . 0 2}$ & $\mathbf{0 . 0 3}$ & $\mathbf{0 . 0 4}$ \\
\hline \multirow{2}{*}{ R } & Neuronal filtering & 38.0081 & 36.6885 & 35.5751 & 34.8253 \\
\cline { 2 - 6 } & Static filtering & 35.1721 & 34.4105 & 33.6808 & 33.2108 \\
\hline \multirow{2}{*}{ G } & Neuronal filtering & 38.4426 & 37.4477 & 36.7999 & 36.1585 \\
\cline { 2 - 6 } & Static filtering & 36.8054 & 36.2199 & 35.8618 & 35.4794 \\
\hline \multirow{2}{*}{ B } & Neuronal filtering & 38.1886 & 37.3777 & 36.7026 & 36.3433 \\
\cline { 2 - 6 } & Static filtering & 36.3987 & 36.0511 & 35.6904 & 35.6383 \\
\hline
\end{tabular}

The result of filtering the image by neuronal and static filtering is shown as follow .The image is currupted by $1 \%$ Salt and pepper noise:

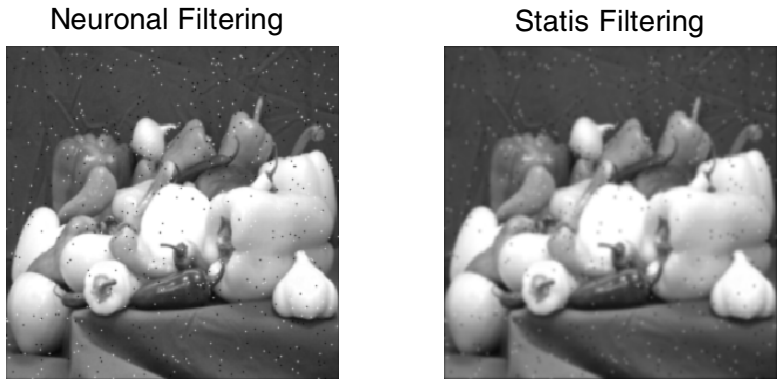

Figure 14. Comparison between neuronal and static filtering method

Actually a low degree of noise remains, the neuronal filtering give an image more clear than the static filtering method. By computing the mean of the three matrix 's psnr an we obtain the final value of the RGB image.

By computing the mean of the three matrix 's psnr an we obtaine the final value of the RGB image as illustrated by the following figure.

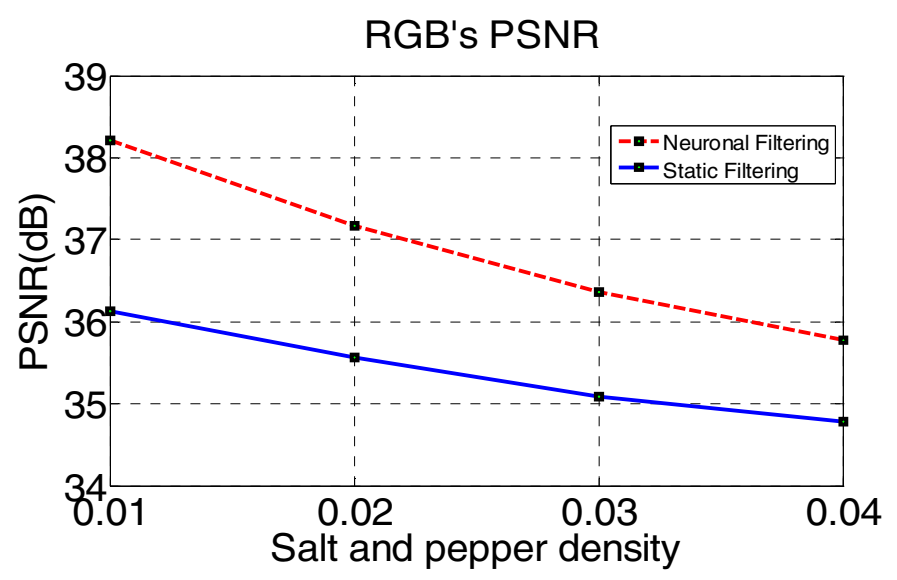

Figure 15. RGB image's PSNR

We have applied the methods above to a variety of test RGB images. All the results of filtering by the dynamic gaussian filter created and supervised by neural networks demonstrate the efficiency of our proposed approach to have a good quality of filtered image.

\section{CONCLUSION}

In this paper an efficient low-pass filter based adaptive Gaussian core is presented. The Gaussian shape is continuously varied over the whole processed image using a neural network. RGB images are used to be filtered with the proposed filter. Many kinds of additive and multiplicative noise are tested and in all the experiences conducted the supervised neural network filter generates better results than the static one. A study is previously conducted to choose the optimal windows size used to be learned by the neural network and the optimal parameters of the Gaussian core. The strength of the filter varies with the image characteristics and noise intensity which engender better result than a fixed size filter. 


\section{References}

[1] C. Tomasi and R. Manduchi, "Bilateral Filtering for Gray and Color Images," Proc. Sixth Int'l Conf. Computer Vision, pp. 839-846, 1998.

[2] F. Luisier, T. Blu, "SURE-LET multichannel image denoising: interscale orthonormal wavelet thresholding," IEEE Transactions on Image Processing, vol. 17, no. 4, pp. 482-492, 2006.

[3] P. M. Narendra. A separable median filter for image noise smoothing. IEEE Trans., PAMI-3(1):20-29, 1981.

[4] K. J. Overton and T. E. Weymouth. A noise reducing preprocessing algorithm. In Proc. IEEE Computer Science Conf on Pattern Recognition and Image Processing, 498-507,1979.

[5] N.Bhoi, S. Meher, "Circular spatial filtering under high noise variance conditions," Computers \& Graphics, Elsevier, vol. 32, no. 5, pp. 568$580,2008$.

[6] M.K. Mihcak, I. Kozintsev, K. Ramchandran, P. Moulin, "Lowcomplexity image denoising based on statistical modeling of wavelet coefficients", IEEE Signal Processing Letters, vol. 6(12), pp.300-303, 1999.

[7] P. Scheunders, S. Backer, "Wavelet denosing of multicomponent images using Gaussian scale mixtures models and a noise-free images as priors," IEEE Transactions on Image Processing, vol. 16, no. 7, pp. 1865-1872, 2007.5, pp. 568-580, 2008.

[8] S. Kim, "PDE-based image restoration: A hybrid model and color image denoising," IEEE Transactions on Image Processing, vol. 15, no. 5, pp. 1163-1170, 2006

[9] N. Lian, V. Zagorodnov, Y. Tan, "Color image denoising using wavelets and minimum cut analysis," IEEE Signal Processing

Letters, vol. 12, no. 11, pp. 741-744, 2005.
[10] S.-J. Chen and J.S. Gibson," Adaptive Restoration of Color Images by a Multichannel Lattice Filter", Mechanical and Aerospace Engineering,University of California, Los Angeles 900951597,Proceedings of the American Control Conference Albuquerque, New Mexico June 1997.

[11] J.P. Cocquerez et S. Philipp, Analyse d.image: filtrage et segmentation, ed. Masson, 1995.

[12] S.J Sangwine and R.E.N Norne, The colour Image Processing Handbook, ed. Chapman \& Hall, 1998.

[13] K.N. Plataniotis \& A.N. Venetsanopoulos, Color Image Processing and Application, ed. Springer, 2000.

[14] J. Chanussot, Approches vectorielles ou marginales pour le traitement d.images multi-composantes, Thèse de l'Université de Savoie soutenue en nov. 1998.

[15] H. Seddik, add E.B. Braiek "Efficient Noise Removing based Optimized Smart Dynamic Gaussian Filter" International Journal Of Computer and Applications, Vol. 51, N=5 ,pp.1-13, 29 August 2012.

[16] S.Meher,"Color Image Denoising with Multi-channel Circular Spatial Filtering" International Conference on Computer Modelling and Simulation, National Institute of Technology,Rourkela-769008, Orissa, India,2010

[17] H.Guan,T.Hara," Color image denoising using e-neighborhood gaussian model", 16-1 Shin ei-cho, Tsuzuki Ward, Yokohama, 224-0035 Japan, September 26-29, 2010,

[18] S.Tebini, H. Seddik and E.B.Braiek, "Optimisation paramétrique neuronale pour un filtre gaussien intelligent appliqué au filtrage d'image", International conférence, CRATT' 2012,Tunis,Tunisie

[19] J. Luc, "Les images numériques, Généralités ", Cours TERI, Diplôme d'Université Assistant àl'Usage des Technologies de l'Information et de la Communication (DU AUTIC) DEUST,Université de Bretagne Occidentale, 2002/2003. 